3. Laporte S, Mismetti P, Décousus $H$, et al; RIETE Investigators. Clinical predictors for fatal pulmonary embolism in 15,520 patients with venous thromboembolism: findings from the Registro Informatizado de la Enfermedad TromboEmbolica venosa (RIETE) Registry. Circulation. 2008;117(13):1711-1716

\section{Common Cold Treatment Using Zinc}

To the Editor The JAMA Clinical Evidence Synopsis on zinc for the common cold by Drs Das and Singh ${ }^{1}$ was a summary of their Cochrane review. ${ }^{2}$ I noticed inconsistencies in the synopsis and so attempted to replicate their data.

In the Evidence Profile section of the synopsis, the authors stated that the number of randomized clinical trials included was "14 therapeutic (lozenges: low-dose $=5$, highdose $=7$, syrup = 2) ... and 2 prophylactic ...," which implies that the reported findings are purely based on randomized comparisons. However, their estimate of 3.43-day shorter colds for high-dose vs low-dose zinc lozenge users appears not to be based on a comparison of randomized groups but rather on an arbitrary selection of 5 high-dose zinc groups from 7 trials compared with 5 low-dose zinc groups from different trials.

There have not been 5 published randomized clinical trials that directly compared high-dose and low-dose zinclozenges. ${ }^{2,3}$ The pairing of the selected high-dose and low-dose zinc groups also appeared arbitrary.

Furthermore, 3 of the 5 low-dose zinc trials had problems in the formulation of their zinc lozenges so that zinc was not released freely, ${ }^{3}$ and therefore, the low dosage of zinc was not the only problem in the low-dose studies.

Das and Singh ${ }^{1}$ stated: "Zinc lozenges were associated with a higher incidence of adverse events compared with placebo (46.5\% for lozenges vs $36.4 \%$ for placebo; number needed to harm [NNH], 10)." This calculation was based on Analysis 2.12 of their Cochrane review. ${ }^{2}$

However, that analysis table counted the placebo group from the study by Turner and Cetnarowski ${ }^{4} 3$ times. A metaanalysis should not count the same randomized participants more than once. The lower rate of adverse events in the placebo group may have been a statistical artifact caused by the triple-counting of a single placebo group.

The negative findings for the low-dose zinc lozenge trials may be caused by the low doses or problems in the lozenge formulations. Therefore, the estimation of zinc lozenge effects should focus on high-dose zinc lozenge studies. Three highdose zinc acetate lozenge trials found that colds were $42 \%(95 \%$ CI, 35\%-48\%) shorter in the zinc groups. ${ }^{3}$

\section{Harri Hemilä, MD, PhD}

Author Affiliation: Department of Public Health, University of Helsinki, Helsinki, Finland.

Corresponding Author: Harri Hemilä, MD, PhD, Department of Public Health University of Helsinki, PO Box 41, Helsinki, Finland FIN-00014 (harri.hemila@helsinki.fi).

Conflict of Interest Disclosures: The author has completed and submitted the ICMJE Form for Disclosure of Potential Conflicts of Interest and none were reported.

1. Das RR, Singh M. Oral zinc for the common cold. JAMA. 2014;311(14):1440-1441. 2. Singh M, Das RR. Zinc for the common cold. Cochrane Database Syst Rev. 2013 6:CD001364
3. Hemilä H. Zinc lozenges may shorten the duration of colds: a systematic review. Open Respir Med J. 2011;5:51-58.

4. Turner RB, Cetnarowski WE. Effect of treatment with zinc gluconate or zinc acetate on experimental and natural colds. Clin Infect Dis. 2000;31(5):1202-1208.

In Reply The findings reported in the JAMA Clinical Evidence Synopsis were based on randomized clinical trials, as was our Cochrane review. ${ }^{1}$ The comparison of the 2 doses $(\geq 75 \mathrm{mg} / \mathrm{d}$ vs $<75 \mathrm{mg} / \mathrm{d}$ ) of zinc was performed as a part of a network metaanalysis, which is a valid technique to assess the comparative effectiveness of interventions among similar study populations that have not been compared directly in randomized clinical trials. ${ }^{2}$

The prerequisite of a network meta-analysis is that the trials should have used the same intervention in the same population and setting, for the same health problem, and measured the same outcome. We therefore selected trials using zinc lozenges that were conducted in the same country (United States), in the same setting (outpatient setting), in the same population (mostly adults), for the same health problem (naturally acquired cold), and for the same outcome (duration of cold). This analysis was not included in the Cochrane review.

We agree with Dr Hemilä's point that 3 of the 5 low-dose zinc trials had problems in the formulation of their zinc lozenges (were nonacetate lozenges) so that zinc was not released freely. However, similar kinds of formulations that released more and less ionic zinc were included in both lowdose and high-dose groups.

There are many possible analytic approaches in a metaanalysis, and we originally chose to triple count adverse event data for 1 study. ${ }^{3}$ A more commonly used method is to combine all relevant intervention groups into a single group, and to combine all relevant control groups into a single control group. ${ }^{4}$

We reanalyzed the data in this way and found a minimal but insignificant difference from the previous calculation (60.2\% for lozenges vs $48.4 \%$ for placebo; number needed to harm [NNH], 9). The conclusion remains the same: "zinc lozenges were associated with a higher incidence of adverse events compared with placebo." This is consistent with findings from published clinical trials on zinc lozenges for the common cold. ${ }^{5}$

Moreover, regarding the study in question, ${ }^{3}$ the authors reported adverse events to be more common in the lozenges group (13\%-19\%; mean, $16 \%$ ) than in the placebo group (10\%).

We agree with Hemilä that the negative findings for the low-dose ( $<75 \mathrm{mg} / \mathrm{d})$ zinc lozenge trials may be related to the low dose or problems in formulations, and the estimation of zinc lozenge effects should focus on trials using high doses $(\geq 75 \mathrm{mg} / \mathrm{d})$ of zinc.

\section{Rashmi Ranjan Das, MD, FCCP Meenu Singh, MD, FCCP}

Author Affiliations: Department of Pediatrics, All India Institute of Medical Sciences, Bhubaneswar, India (Das); Department of Pediatrics, Post-Graduate Institute of Medical Education and Research, Chandigarh, India (Singh).

Corresponding Author: Meenu Singh, MD, FCCP, Department of Pediatrics, Post-Graduate Institute of Medical Education and Research, Chandigarh-160012, India (meenusingh4@gmail.com). 
Conflict of Interest Disclosures: The authors have completed and submitted the ICMJE Form for Disclosure of Potential Conflicts of Interest and none were reported.

1. Singh M, Das RR. Zinc for the common cold. Cochrane Database Syst Rev. 2013;6(6):CD001364.

2. Chambers JD, Naci H, Wouters OJ, et al. An assessment of the methodological quality of published network meta-analyses: a systematic review. PLoS One. 2015;10(4):e0121715.

3. Turner RB, Cetnarowski WE. Effect of treatment with zinc gluconate or zinc acetate on experimental and natural colds. Clin Infect Dis. 2000;31(5):1202-1208.

4. Higgins JPT, Deeks JJ, Altman DG, eds. How to include multiple groups from 1 study. In: Higgins JPT, Green S, eds. Cochrane Handbook for Systematic Reviews of Interventions. London, England: Cochrane Collaboration; 2011:chap 16.5.4.

5. Macknin ML, Piedmonte $M$, Calendine $C$, Janosky J, Wald E. Zinc gluconate lozenges for treating the common cold in children: a randomized controlled trial. JAMA. 1998;279(24):1962-1967.

\section{CORRECTION}

Incorrect Dose in Figure: In the Original Contribution entitled "Effect of Patiromer on Serum Potassium Level in Patients With Hyperkalemia and Diabetic Kidney Disease: The AMETHYST-DN Randomized Clinical Trial" published in the July 14, 2015, issue of JAMA, ${ }^{1}$ an incorrect study drug dose appeared in a figure. In Figure 2, the starting patiromer dose reported as 16.2 in the $\mathrm{x}$-axis for both stratum 1 and stratum 2 in both the left and the right plots should have appeared as 16.8. This article was corrected online.

1. Bakris GL, Pitt B, Weir MR, et al; AMETHYST-DN Investigators. Effect of patiromer on serum potassium level in patients with hyperkalemia and diabetic kidney disease: the AMETHYST-DN randomized clinical trial. JAMA. 2015;314(2): 151-161.

Wording Errors in the Text: In the Viewpoint entitled "Innovations of the Americans With Disabilities Act: Confronting Disability Discrimination in Employment" published in the June 9, 2015, issue of JAMA, ${ }^{1}$ the article should have stated more clearly that the pre-Americans with Disabilities Act Amendments Act (ADAAA) requirements for establishing coverage under the "regarded as" part of the defini- tion are no longer required under the ADAAA. Under the ADAAA, an individual is regarded as having a disability if he or she has been subject to discrimination "because of an actual or perceived physical or mental impairment whether or not the impairment limits or is perceived to limit a major life activity." This article was corrected online.

1. Rothstein MA. Innovations of the Americans with Disabilities Act: confronting disability discrimination in employment. JAMA. 2015;313(22):2221-2222.

\section{Guidelines for Letters}

Letters discussing a recent JAMA article should be submitted within 4 weeks of the article's publication in print. Letters received after 4 weeks will rarely be considered. Letters should not exceed 400 words of text and 5 references and may have no more than 3 authors. Letters reporting original research should not exceed 600 words of text and 6 references and may have no more than 7 authors. They may include up to 2 tables or figures but online supplementary material is not allowed. All letters should include a word count. Letters must not duplicate other material published or submitted for publication. Letters not meeting these specifications are generally not considered. Letters being considered for publication ordinarily will be sent to the authors of the JAMA article, who will be given the opportunity to reply. Letters will be published at the discretion of the editors and are subject to abridgement and editing. Further instructions can be found at http://jama.com/public /InstructionsForAuthors.aspx. A signed statement for authorship criteria and responsibility, financial disclosure, copyright transfer, and acknowledgment and the ICMJE Form for Disclosure of Potential Conflicts of Interest are required before publication. Letters should be submitted via the JAMA online submission and review system at http: //manuscripts.jama.com. For technical assistance, please contact jama-letters@jamanetwork.org.

Section Editor: Jody W. Zylke, MD, Deputy Editor 